\title{
Elimination of cross talk in waveguide intersections
}

\author{
Steven G. Johnson \\ Department of Physics, Massachusetts Institute of Technology, Cambridge, Massachusetts 02139
}

Christina Manolatou

Department of Electrical Engineering and Computer Science, Massachusetts Institute of Technology, Cambridge, Massachusetts 02139

Shanhui Fan, Pierre R. Villeneuve, and J. D. Joannopoulos

Department of Physics, Massachusetts Institute of Technology, Cambridge, Massachusetts 02139

\section{H. A. Haus}

Department of Electrical Engineering and Computer Science, Massachusetts Institute of Technology, Cambridge, Massachusetts 02139

Received August 26, 1998

\begin{abstract}
We present general criteria for crossing perpendicular waveguides with nearly $100 \%$ throughput and $0 \%$ cross talk. Our design applies even when the waveguide width is of the order of the wavelength. The theoretical basis for this phenomenon is explained in terms of symmetry considerations and resonant tunneling and is then illustrated with numerical simulations for both a two-dimensional photonic crystal and a conventional highindex-contrast waveguide crossing. Cross-talk reduction by up to 8 orders of magnitude is achieved relative to unmodified crossings. (c) 1998 Optical Society of America

OCIS codes: $\quad 230.3120,230.3990,130.3120,130.1750,230.7370$.
\end{abstract}

The ability to intersect waveguides is crucial in constructing integrated optical circuits, owing to the desire for complex systems involving multiple waveguides. We present a novel theoretical framework for achieving low cross talk and high throughput in perpendicular intersections, based on symmetry considerations that can be systematically applied to any optical system. The transmission spectra are predictable without detailed calculations and are robust under perturbations that do not break the symmetry. The focus of this design is the elimination of cross talk; additional considerations are required for maximization of throughput when radiation losses are present. We illustrate the theory with two example systems, a two-dimensional photonic crystal and a conventional dielectric-waveguide intersection, and show how one can produce efficient crossing, by assembling well-understood elements that are independently tuned. In contrast, previous studies of waveguide intersections $\mathrm{s}^{1,2}$ have lacked general principles that could be applied a priori to diverse systems. Moreover, they have typically been concerned with shallow-angle crossings for wavelengths many times smaller than the waveguide width. Although perpendicular crossings in such systems exhibit negligible cross talk, the cross talk is close to $10 \%$ when the waveguide width is of the order of half a wavelength (as seen below). Our design applies even when the waveguide width is small, permitting single-mode waveguides with optimal miniaturization. Simulations of our structures show peak throughputs of nearly unity, with cross talk as low as $10^{-9}$.

The fundamental idea is to consider coupling of the four branches, or ports, of the intersection in terms of a resonant cavity at the center. If the resonant modes that are excited from the input port can be prevented by symmetry from decaying into the transverse ports, then cross talk is eliminated and the system reduces to the well-known phenomenon of resonant tunneling through a cavity. This situation can be achieved by means of the following conditions (see also Fig. 1):

- Each waveguide must have a mirror symmetry plane through its axis and perpendicular to the other waveguide and have a single guided mode in the frequency range of interest. This mode will be either even or odd with respect to the mirror plane.

- The center of the intersection must be occupied by a resonant cavity that is symmetric with respect to the mirror planes of both waveguides.

- Two resonant modes must exist in the cavity, each of which is even with respect to one waveguide's mirror

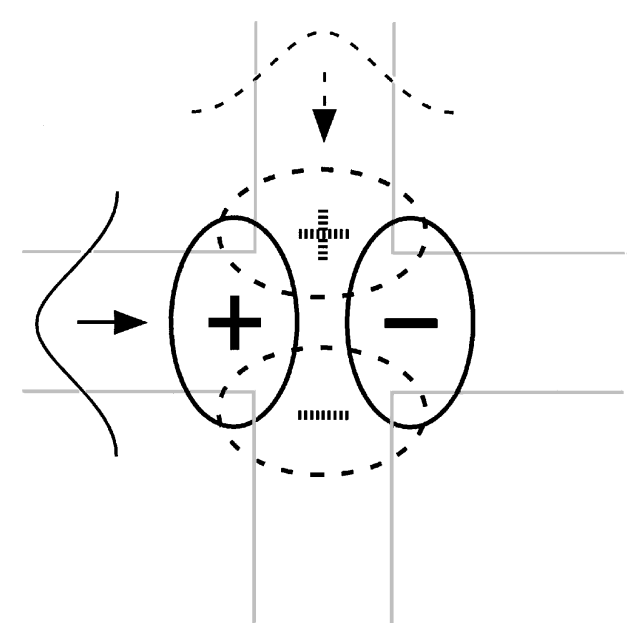

Fig. 1. Abstract diagram of symmetry requirements for waveguide crossing, showing waveguide mode profiles and resonant-cavity mode contours. By symmetry, the solidline modes cannot couple with the dashed-line modes and vice versa. 
plane and odd with respect to the other. These modes should be the only resonant modes in the frequency range of interest.

If these requirements are satisfied, then each resonant state will couple to modes in just one waveguide and be orthogonal to modes in the other waveguide, as shown in Fig. 1. (For simplicity, we depict a lowestorder even waveguide mode.) Therefore, under the approximation that the ports couple to one another only through the resonant cavity, cross talk will be prohibited. The transmission to the output port is described by resonant tunneling, and one can use coupled-mode theor $\mathrm{y}^{3}$ to show that the throughput spectrum will be a Lorentzian curve peaked at unity on resonance. The width of the Lorentzian is given by the inverse of the cavity's quality factor $Q$, which is proportional to the lifetime of the resonance mode.

Perfect throughput will not be attained, however, owing to a combination of two effects: direct coupling and radiation loss. First, the input port can directly couple with the transverse ports, resulting in cross talk, which can be decreased arbitrarily but at the expense of the throughput bandwidth. Making the cavity larger reduces direct coupling, since this increases the distance between the localized waveguide modes. The drawback to increased cavity size is that it can either create extraneous resonance modes or, as in the case of the photonic crystal cavities described below, increase the $Q$ of the resonance. Second, many systems suffer from losses that are due to radiation or other mechanisms. If these losses do not couple strongly to the transverse waveguide, then their primary effect will be to decrease throughput and increase reflection. ${ }^{3}$

The required parity of the cavity modes is easy to achieve in practice, owing to the fact that a perpendicular intersection of identical waveguides typically has $C_{4 v}$ symmetry (the symmetry group of the square). In this case, any nonaccidental degenerate modes (such as many higher-order modes) will necessarily be a pair having the requisite symmetry from Fig. 1, as that is the only two-dimensional irreducible representation of $C_{4 v}{ }^{4}$

A realization of the intersection design can be made in a photonic crystal, ${ }^{5}$ for which the bandgap in the bulk material prevents any radiation losses. For ease of computation we use a two-dimensional crystal consisting of a square lattice of dielectric rods in air. The rods have lattice constant $a$, radius $0.2 a$, and dielectric constant 11.56. This structure prohibits propagation of TM light (in-plane magnetic field) in the frequency range 0.286 to $0.421 \mathrm{c} / a .^{5}$ Removing a row or column of rods produces a waveguide. ${ }^{5}$ Increasing the radius of a single rod to $0.33 a$ creates a resonant cavity, leading to doubly degenerate modes of the requisite symmetry with a frequency of $0.361 c / a$ (i.e., a wavelength in air of $2.77 a){ }^{6}$

These elements are then combined into a intersection, and we simulate the collective behavior of several structural variations (Fig. 2) using a finite-difference time-domain simulation. ${ }^{7}$ In each case we send a wide-spectrum TM Gaussian pulse to the input port. The fractional power transmission is then evaluated as a function of frequency for the output port and one of the transverse ports, yielding the throughput and the cross talk, respectively. The resulting transmission spectra are shown in Fig. 3. Up to $5 \%$ of each throughput and cross-talk value can be due to reflections from the boundary of the simulation, which is why the peak throughput in cases (c) and (d) shown in Fig. 2 appears to exceed unity.

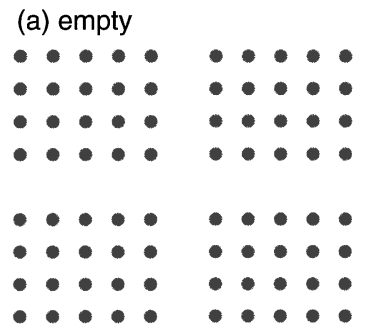

(c) $3 \times 3$

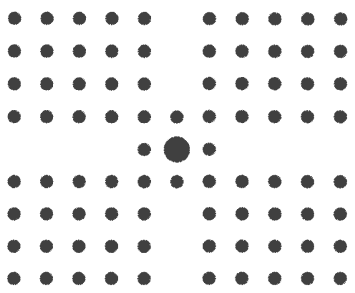

(b) $5 \times 5$

$\bullet \bullet \bullet \bullet \bullet \bullet$

- $\bullet \bullet \bullet$

- $\bullet \bullet \bullet \bullet$

- $\bullet \bullet \bullet \bullet$

-

$\bullet \bullet \bullet \bullet \bullet \bullet \bullet$

- $\bullet \bullet \bullet \bullet$

$\bullet \bullet \bullet \bullet \bullet \bullet \bullet \bullet$

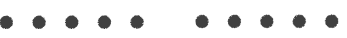

(d) $1 \times 1$

$\bullet \bullet \bullet \bullet \bullet \bullet$

$\bullet \bullet \bullet \bullet \bullet \bullet \bullet$

$\bullet \bullet \bullet \bullet \bullet \bullet \bullet \bullet$

$\bullet \bullet \bullet \bullet \bullet \bullet \bullet \bullet$

$\bullet \bullet \bullet \bullet \bullet \bullet \bullet$

$\bullet \bullet \bullet \bullet \bullet \bullet$

$\bullet \bullet \bullet \bullet \bullet \bullet$

- $\bullet \bullet \bullet \bullet \bullet$

Fig. 2. Waveguide intersections in a two-dimensional photonic crystal consisting of a square lattice of dielectric rods in air.

(a)

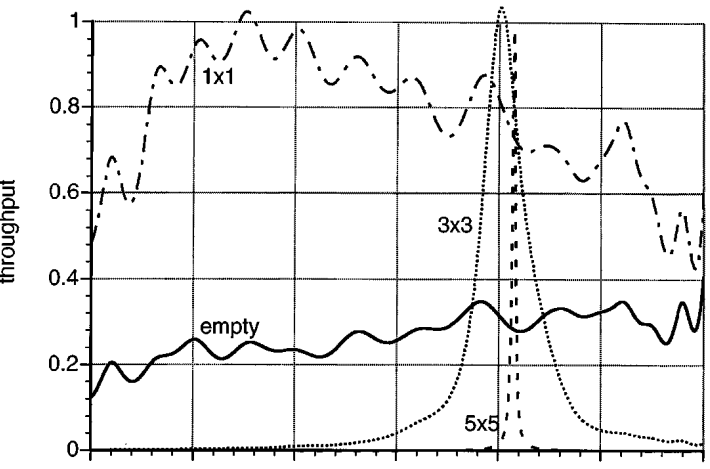

(b)

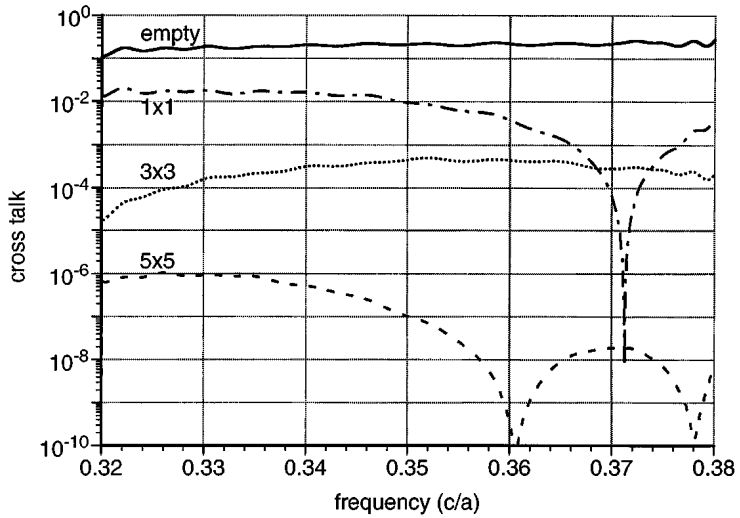

Fig. 3. TM transmission spectra for the four intersections shown in Fig. 2: (a) throughput from the input port to the output port and (b) cross talk from the input port to one of the transverse ports. 


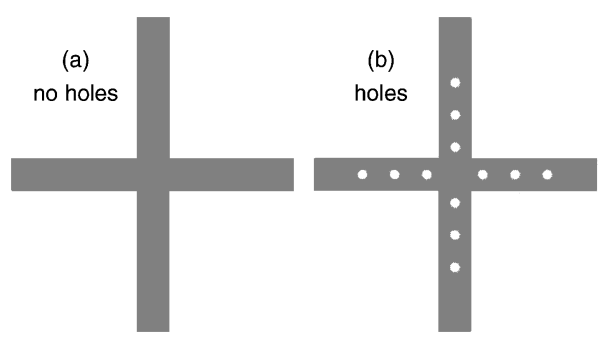

Fig. 4. Intersection of two-dimensional, high-indexcontrast waveguides in air, both (a) without holes and (b) with air holes added to create a resonant cavity by use of a photonic bandgap.

(a)

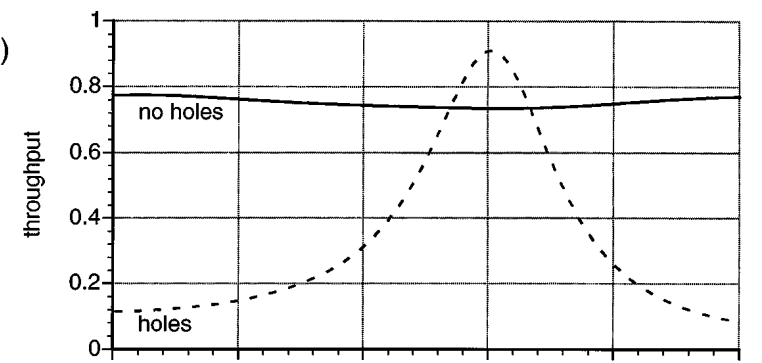

(b)

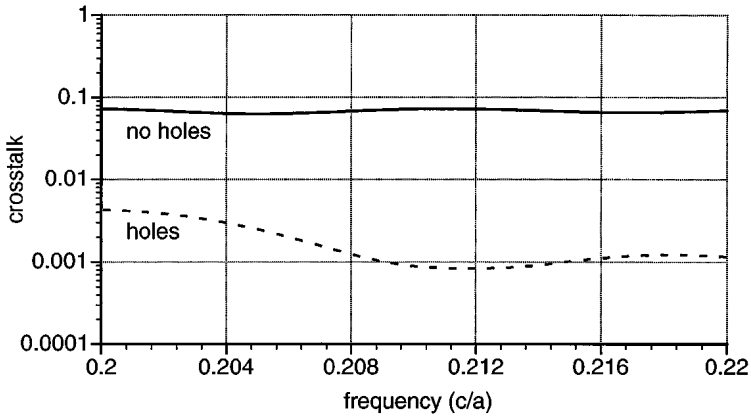

Fig. 5. TE transmission spectra for the two intersections shown in Fig. 4.

As predicted, the throughput for cases (b) and (c) is a Lorentzian centered on the resonance frequency (which varies owing to the finite size of the cavity). At their resonance frequencies, (b) and (c) achieve cross talk of $5 \times 10^{-10}$ and $4 \times 10^{-4}$, respectively. In case (d) there are no photonic crystal layers separating the central rod from the waveguide, and confinement is due only to index contrast. The cavity confinement is so weak that coupled-mode theory is no longer accurate. Even so, case (d) reaches nearly $100 \%$ throughput with a cross talk at the same frequency of $1.8 \%$. In contrast, both the throughput and the cross talk for the empty intersection lie in the 20-40\% range.

Both the cross talk and the resonance $Q$ derive from the exponential decay of light through the bulk photonic crystal. Because the cross talk must tunnel through twice as many crystal layers as the resonance decay, the cross talk should be approximately $Q^{-2}$. Looking at Fig. 3(b), we see that this estimate for the cross talk can be seen to be valid to within approximately an order of magnitude, corresponding to $Q$ of roughly 1000, 100, and 10 for cases (b), (c), and (d), respectively. Here, we do not consider the additional resonance minima that are apparent in the cross talk at some frequencies, which are independent of the throughput resonance and are due to a destructive-interference phenomenon that is still under investigation.

The same principles of intersection design can be applied to other systems, such as conventional dielectric waveguide crossings. In particular, we consider an intersection of high-index-contrast waveguides in air, of width $a$ and dielectric constant 11.56, as shown in Fig. 4(a). As shown in Fig. 4(b), we modified the crossing to include a resonant cavity by introducing a periodic sequence of air holes on each branch of the intersection, creating a bandgap in the TE guided modes of the waveguides. ${ }^{8}$ The holes have radius $0.2 a$ and period $a$. A defect center-to-center separation of $2.2 a$ produces a pair of doubly degenerate, $p$-like modes with the desired symmetry at a frequency of $0.22 c / a$ (i.e., a wavelength in the dielectric of $1.3 a$ ).

As before, we used a two-dimensional simulation to evaluate the throughput and cross talk of these two structures, this time with TE-polarized light. The results are shown in Fig. 5. Not only does the resonant cavity increase the throughput from $75 \%$ to more than $90 \%$, but also the cross talk is decreased by 2 orders of magnitude, from $7 \%$ to $0.08 \%$. The resonance $Q$ is close to 40 and, as before, the cross talk can be estimated by use of $Q^{-2}$. The main difference between the behavior of this crossing and the one shown in Fig. 2 is that there are now radiation losses from scattering off the intersection, resulting in lower throughput.

The principles described in this Letter also apply to three-dimensional systems and are not limited to photonic-crystal cavities. Further refinements could include tuning the intersection in Fig. 4 to reduce radiation losses or using a system of resonators to flatten the resonant peak. ${ }^{9}$

This work was supported in part by the Materials Research Science and Engineering Center program of the National Science Foundation under award DMR9400334. S. G. Johnson gratefully acknowledges support from the National Defense Science and Engineering Graduate Fellowship Program. S. G. Johnson's e-mail address is stevenj@alum.mit.edu.

\section{References}

1. K. Aretz and H. Bülow, Electron. Lett. 25, 730 (1989).

2. M. G. Daly, P. E. Jessop, and D. Yevick, J. Lightwave Technol. 14, 1695 (1996).

3. H. A. Haus, Waves and Fields in Optoelectronics (Prentice-Hall, Englewood Cliffs, N.J., 1984), Chap. 7.

4. See, e.g., M. Tinkham, Group Theory and Quantum Mechanics (McGraw-Hill, New York, 1964).

5. J. D. Joannopoulos, P. R. Villeneuve, and S. Fan, Nature (London) 386, 143 (1997).

6. P. R. Villeneuve, S. Fan, and J. D. Joannopoulos, Phys. Rev. B 54, 7837 (1996).

7. See, e.g., K. S. Kunz and R. J. Luebbers, The FiniteDifference Time-Domain Methods (CRC, Boca Raton, Fla., 1993).

8. S. Fan, J. N. Winn, A. Devenyi, J. C. Chen, R. D. Meade, and J. D. Joannopoulos, J. Opt. Soc. Am. B 12, 1267 (1995).

9. See, e.g., H. A. Haus and Y. Lai, IEEE J. Quantum Electron. 28, 205 (1992). 